\title{
Zone-Doubling Technique to Produce Ultrahigh-Resolution X-Ray Optics
}

\author{
K. Jefimovs, ${ }^{1,2}$ J. Vila-Comamala, ${ }^{3}$ T. Pilvi, ${ }^{4}$ J. Raabe, ${ }^{1}$ M. Ritala, ${ }^{4}$ and C. David ${ }^{1}$ \\ ${ }^{1}$ Paul Scherrer Institut, CH-5232 Villigen PSI, Switzerland \\ ${ }^{2}$ EMPA, CH-8600 Dübendorf, Switzerland \\ ${ }^{3}$ Laboratori de Llum Sincrotró, E-08193 Bellaterra, Spain \\ ${ }^{4}$ Laboratory of Inorganic Chemistry, University of Helsinki, FI-00014 Helsinki, Finland
}

(Received 18 September 2007; published 28 December 2007)

\begin{abstract}
A method for the fabrication of ultrahigh-resolution Fresnel zone plate lenses for x-ray microscopy is demonstrated. It is based on the deposition of a zone plate material (Ir) onto the sidewalls of a prepatterned template structure ( $\mathrm{Si}$ ) using an atomic layer deposition technique. This results in a doubling of the effective zone density, thus improving the achievable resolution of x-ray microscopes. Test structures with lines and spaces down to $15 \mathrm{~nm}$ were resolved in a scanning transmission $\mathrm{x}$-ray microscope at $1 \mathrm{keV}$ photon energy.
\end{abstract}

$\mathrm{X}$-ray microscopy is an outstanding technique for the investigation of both inorganic and biological samples, as it possesses high spatial resolution, good penetration capability, combined with specific contrast mechanisms that can provide information on the elemental and even chemical composition of a specimen. Disciplines such as biology, materials science, chemistry, and environmental science are benefiting from the progress achieved in x-ray microscopes in recent years. The theoretically attainable resolution limit is much better than that of visible light microscopes, due to the 100-1000 times shorter wavelength. However, this limit has not been reached yet due to the limitations in the fabrication of the diffractive Fresnel zone plate (FZP) lenses used for imaging. In the hard $\mathrm{x}$-ray regime sub-50 $\mathrm{nm}$ resolution has been reached $[1,2]$. In the soft $\mathrm{x}$-ray regime, the daily working resolution is around $30 \mathrm{~nm}$, while the smallest lines resolved are $15 \mathrm{~nm}$ wide [3].

There is an intimate relationship between the spatial resolution of a FZP-based microscope and the outermost zone width of the FZP, the two of them being essentially comparable. Electron-beam lithography tools, which are typically used to generate FZPs, are capable of writing with nanometer spot sizes and position accuracies. However, the obtainable structure sizes are determined by the range of secondary electrons created in the resist layer, which has a particularly detrimental consequence when writing dense patterns of lines such as gratings or FZPs. An approach has recently been reported which overcomes this nanofabrication limit by use of an overlay technique [3]. It is based on two separate exposure steps of complementary patterns to reduce the line density in each individual step. A FZP with $15 \mathrm{~nm}$ outermost zone width was demonstrated with this technique. Extremely small alignment tolerances of only a few nanometers are required between the two exposures, making the technique inherently complicated and resulting in a low fabrication yield.
Here we report on a powerful and, at the same time, much simpler nanofabrication method that overcomes the difficulty of high feature density patterning without any need for alignment. The method is very reproducible, as it only requires a single lithography step, and allows the fabrication of structures with extremely high aspect ratios, which increases the efficiency of ultrahigh-resolution optics. It is based on a deposition of a thin layer of a high refractive index material onto the sidewalls of a template structure made of a low-index material. This leads to a doubling of the effective line density of the deposited

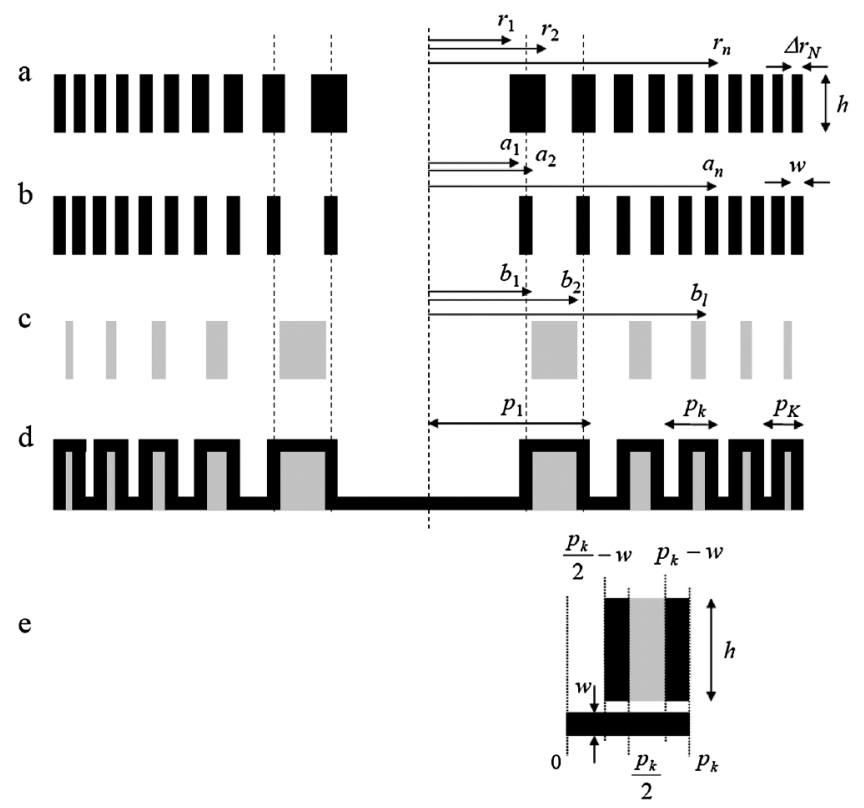

FIG. 1. (a) Schematic drawing of a Fresnel zone plate, (b) Fresnel zone plate with a constant width of the even zones equal to $w$, (c) low refractive index template structure, (d) low refractive index template structure coated by high refractive index zone plate material, and (e) fragment of the zone plate used for calculation of the local diffraction efficiency. 
material compared to that of the template [4,5], which improves the resolution of fabricated FZP by a factor of two. The method requires highly conformal deposition of the high refractive index material with accurately controlled film thickness. Because of its unique self-limiting growth mechanism, atomic layer deposition [6] is the best choice for this purpose and was employed here. Figure 1 illustrates how this technique can be applied to the fabrication of a FZP.

To derive the geometry of such a device and that of the required template, we first consider a normal FZP having focal distance $f$ at a wavelength $\lambda$ [Fig. 1(a)] with the radius of the $n$th zone boundary $r_{n}$ described by the wellknown equation [7]

$$
r_{n}^{2}=n f \lambda+\frac{1}{4} n^{2} \lambda^{2}, \quad \text { for } \quad n=1,2,3, \ldots
$$

We define the first zone $\Delta r_{1}$ as the disk around the center of the FZP to the first zone boundary $\left(\Delta r_{1}=r_{1}\right)$, the second zone as the ring between $r_{1}$ and $r_{2}\left(\Delta r_{2}=r_{2}-\right.$ $\left.r_{1}\right)$, and so on $\left(\Delta r_{n}=r_{n}-r_{n-1}\right)$. Let us consider a zone plate, where the width of the even zones is fixed and equal to $w$ [see Fig. 1(b)]. To a good approximation the zone boundaries $a_{n}$ of such a structure are given by

$$
a_{n}=\sqrt{\frac{r_{n}^{2}+r_{n+1}^{2}}{2}}-\frac{w}{2}, \quad \text { for } n=1,3,5, \ldots
$$

and

$$
a_{n}=\sqrt{\frac{r_{n-1}^{2}+r_{n}^{2}}{2}}+\frac{w}{2}, \quad \text { for } n=2,4,6, \ldots
$$

A FZP shown in Fig. 1(b) can be created by coating the sidewalls of a template structure [Fig. 1(c)], and zone boundaries $b_{l}$ are given by

$$
b_{l}=\sqrt{\frac{r_{2 n-1}^{2}+r_{2 n}^{2}}{2}}-i^{2 n} \frac{w}{2}, \quad \text { for } \quad n=1,2,3, \ldots
$$

where $i^{2 n}$ converts to -1 for odd and to +1 for even values of $n$. During the coating process the high-index material will also be deposited on the ridges of the template and in the grooves between them, as illustrated in Fig. 1(d). The effect on $\mathrm{x}$ rays passing through the device is identical to the one for the structure shown in Fig. 1(e), consisting of the sum of the structures $b$ and $c$ and a homogeneous layer of high-index material.

Since the design of the FZP shown in Fig. 1(d) is different from that of a "conventional" FZP structure, the diffraction efficiency (DE) is expected to change accordingly. In order to optimize the FZP structure for given material parameters, we derive in the following the relations required to calculate the DE. The deposited zone plate material should have a strong interaction with the $\mathrm{x}$ rays passing through the device, i.e., a high refractive index, whereas the template should affect the radiation as little as possible. Silicon and polymers have a low refrac- tive index over a wide range of energies and there exist well-developed methods for their nanostructuring, which makes them well suited as template material. The choice of the high refractive index material greatly depends on the energy to be used. We performed several scalar calculations of the DE for different material pairs and different energies in the thin element approximation [8]. In the x-ray range, the complex refractive index of the material is $n=$ $1-\delta-i \beta$, where $\delta$ accounts for the phase shift and $\beta$ for the absorption [9]. For a good approximation, we can assume that the high refractive index zones are locally equidistant; i.e., the width of a low refractive index zone is equal to the width of the closest open zone. In order to correctly account for the effects caused by the low-index template we need to regard a full period as containing two high-index structures, i.e., four zones [see Fig. 1(e)]. In analogy to the equations derived by Kirz [9], we express the flux in $m$ th diffraction order due to the $k$ th local period of a template coated by a zone plate material as

$$
I_{k}=\left|A_{1}+A_{2}+A_{3}+A_{4}\right|^{2}
$$

where

$$
A_{1}=\frac{C}{p_{k}} \exp \left(-\frac{2 \pi}{\lambda} \beta_{\text {high }} w\right) \int_{0}^{p_{k} / 2-w} \exp \left(-2 \pi i \frac{m x}{p_{k}}\right) d x
$$

$$
\begin{aligned}
A_{2}= & \frac{C}{p_{k}} \exp \left(-\frac{2 \pi}{\lambda} \beta_{\text {high }} w\right) \exp \left(-\frac{2 \pi}{\lambda} \beta_{\text {high }} h\right) \\
& \times \exp \left(-\frac{2 \pi i}{\lambda} \delta_{\text {high }} h\right) \int_{p_{k} / 2-w}^{p_{k} / 2} \exp \left(-2 \pi i \frac{m x}{p_{k}}\right) d x,
\end{aligned}
$$

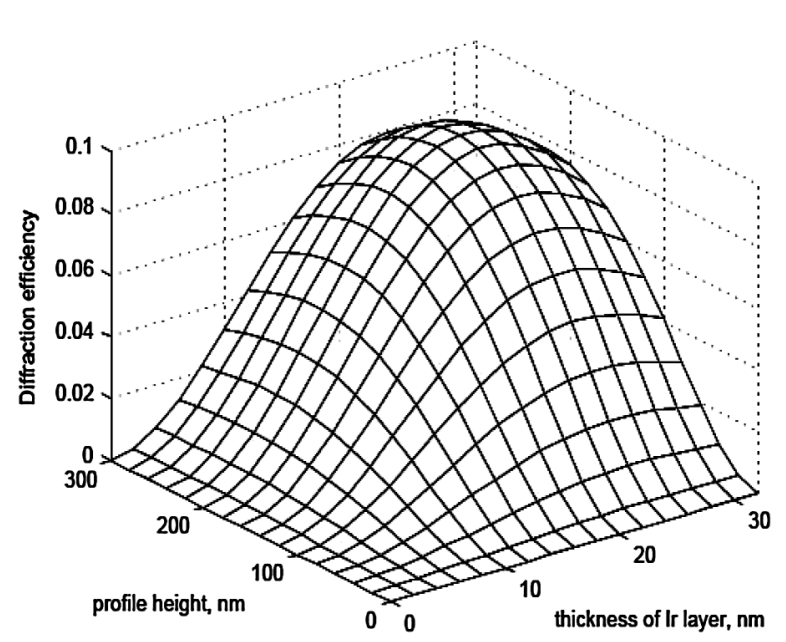

FIG. 2. Diffraction efficiency of a zone plate consisting of a $\mathrm{Si}$ template with $80 \mathrm{~nm}$ outermost period, coated with an Ir layer [see Figs. 1(d) and 1(e)], as a function of the profile height and the thickness of the Ir layer at $1 \mathrm{keV}$ photon energy. 


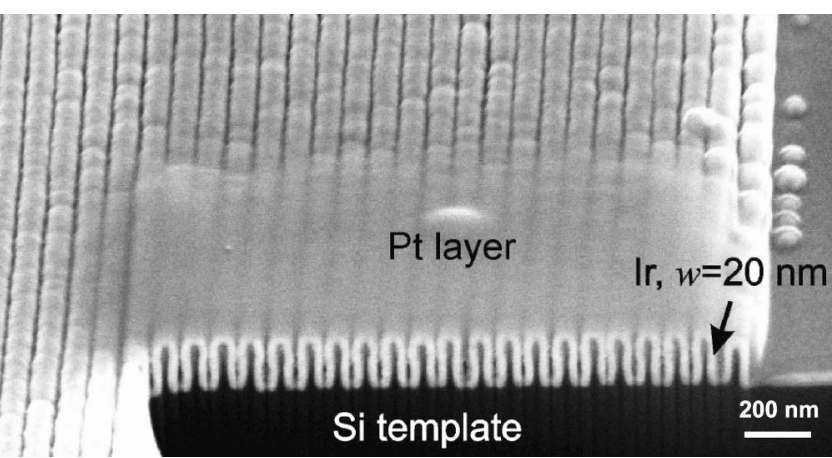

FIG. 3. SEM micrograph of an Ir zone plate with an outermost zone width of $20 \mathrm{~nm}$. A small area of the zone plate was coated with a thin Pt layer in order to ensure a clean cross sectioning by focused ion beam milling.

$$
\begin{aligned}
A_{3}= & \frac{C}{p_{k}} \exp \left(-\frac{2 \pi}{\lambda} \beta_{\text {high }} w\right) \exp \left(-\frac{2 \pi}{\lambda} \beta_{\text {low }} h\right) \\
& \times \exp \left(-\frac{2 \pi i}{\lambda} \delta_{\text {low }} h\right) \int_{p_{k} / 2}^{p_{k}-w} \exp \left(-2 \pi i \frac{m x}{p_{k}}\right) d x
\end{aligned}
$$

$$
\begin{aligned}
A_{4}= & \frac{C}{p_{k}} \exp \left(-\frac{2 \pi}{\lambda} \beta_{\text {high }} w\right) \exp \left(-\frac{2 \pi}{\lambda} \beta_{\text {high }} h\right) \\
& \times \exp \left(-\frac{2 \pi i}{\lambda} \delta_{\text {high }} h\right) \int_{p_{k}-w}^{p_{k}} \exp \left(-2 \pi i \frac{m x}{p_{k}}\right) d x
\end{aligned}
$$

are the amplitudes contributed by the four different zones of the $k$ th local period $p_{k}$. Index $k$ corresponds to $k=2 l=$ $4 n$. The indices "high" and "low" refer to high and low refractive index material, respectively. $C^{2}=I_{\text {inc }}$ is the incident flux. The first exponential term in all four expressions corresponds to the absorption in the uniform layer [see Fig. 1(e)] of the high refractive index material. The second and the third terms correspond to the absorption and the phase shift by a certain zone of a height $h$ of the zone plate. Fresnel integrals represent the path difference of the beam passing through a certain zone to a focal plane. As each period contains two high-index structures, the DE of the first order should be calculated for $m=2$. Since in the case of round FZP the area of each period to a good approximation is the same for all values of $k$, the DE of the FZP can be calculated as the average of the local efficien- cies:

$$
\eta_{\mathrm{tot}}=\frac{1}{K} \sum_{k=1}^{K} I_{k} / I_{\mathrm{inc}}
$$

where $K$ is the number of periods in a template structure.

Using these equations one can perform a numerical optimization of the DE as a function of deposited layer thickness $w$ for a given set of materials, photon energy, and template height $h$. In contrast to the case of a conventional FZP as described by Kirz [9] [Fig. 1(a)], the efficiency of the FZP shown in Fig. 1(d) also depends on the absolute width of the outermost zone structures. Only for the case of zero absorption $\beta=0$ the first exponential term in Eqs. (5)-(8) is equal to unity and a zone-widthindependent solution can be given. It results in a maximum DE of $32 \%$ of the FZP shown in Fig. 1(b). In comparison, the theoretical limit of DE of a classical binary RayleighWood phase reversal zone plate is $40.5 \%$ [9]. Therefore, by choosing a proper combination of the template and the zone plate materials, the FZP shown in Fig. 1(d) could reach an efficiency of up to 4/5 of that of the standard FZP.

We have chosen $\mathrm{Si}$ and $\mathrm{Ir}$ as low and high refractive index materials, respectively, because of the large difference between their complex refractive indices [10] and the available fabrication possibilities. The result of DE calculations for $1 \mathrm{keV}$ x-ray energy and a FZP with $40 \mathrm{~nm}$ outermost period of the Ir structures is shown in Fig. 2. The values of the maximum obtainable diffraction efficiency (peak value in Fig. 2) as well as the corresponding profile heights and thicknesses of the Ir layer for different energies are summarized in Table I. For comparison we also present values of the DE for the FZP shown in Fig. 1(b) and the classical FZP shown in Fig. 1(a). As can be seen from the table, the effect of the presence of the template material and the Ir absorbing layer between zones is negligible for hard X-ray energies. However, the structure height required to obtain efficient focusing is significantly less for lower photon energies, thus smaller outermost zone widths can be achieved. As a compromise between the efficiency and the resolution, we have chosen $1 \mathrm{keV}$ energy for "a proof of principle" experiment.

The template structure was patterned by electron-beam lithography using a high resolution negative tone resist

\begin{tabular}{|c|c|c|c|c|c|c|c|c|c|}
\hline \multirow[b]{2}{*}{$\mathrm{X}$-ray energy $(\mathrm{eV})$} & \multicolumn{3}{|c|}{ Figure 1(a) } & \multicolumn{3}{|c|}{ Figure 1(b) } & \multicolumn{3}{|c|}{ Figure 1(d) } \\
\hline & $h(\mathrm{~nm})$ & $\Delta r_{N}(\mathrm{~nm})$ & $\mathrm{DE}(\%)$ & $h(\mathrm{~nm})$ & $w(\mathrm{~nm})$ & $\mathrm{DE}(\%)$ & $h(\mathrm{~nm})$ & $w(\mathrm{~nm})$ & $\mathrm{DE}(\%)$ \\
\hline 500 & 150 & & 12 & 151 & 25.9 & 9.4 & 135 & 21.7 & 3.5 \\
\hline 1000 & 215 & 20 & 17 & 218 & 25.9 & 13.5 & 225 & 24.8 & 9.1 \\
\hline 8000 & 1350 & & 31 & 1350 & 25.9 & 24.6 & 1400 & 25.6 & 22.9 \\
\hline
\end{tabular}
(calixarene) and transferred into a $\mathrm{Si}$ membrane by reactive ion etching using an intermediate $\mathrm{Cr}$ mask (the details of

TABLE I. Maximum obtainable diffraction efficiencies of different types of zone plates shown in Fig. 1. The calculations are made for iridium as zone plate material and silicon as template material. The outermost period of the $\mathrm{Ir}$ structures is $40 \mathrm{~nm}$. 

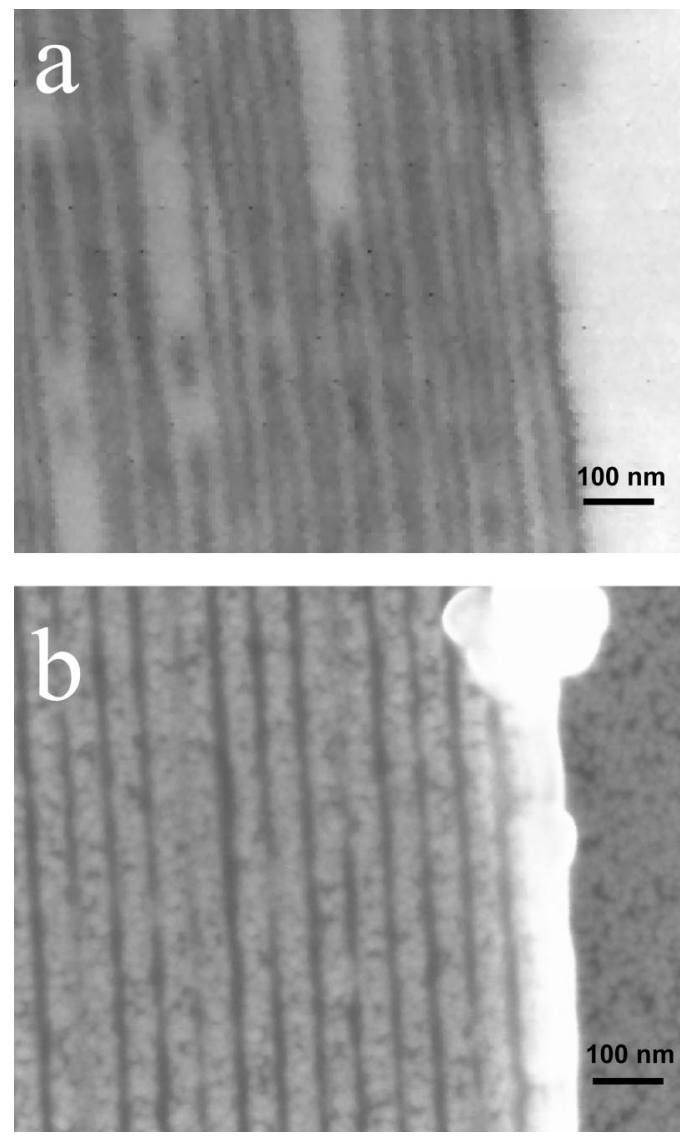

FIG. 4. STXM (a) and SEM (b) images of the same region of a test structure with $15 \mathrm{~nm}$ lines and spaces. The SEM image shows two times larger periodicity $(60 \mathrm{~nm})$, as electrons do not penetrate the Ir layer deposited on top of the Si template lines. $\mathrm{X}$ rays, however, penetrate through both the Si template and the thin Ir layer, revealing the Ir lines deposited onto the sidewalls of the Si template.

the process will be published elsewhere). The height of the obtained Si template structures was $170 \mathrm{~nm}$, and the diameter of the template was $100 \mu \mathrm{m}$ with an outermost period of $80 \mathrm{~nm}$. The etched template was coated with $20 \mathrm{~nm}$ Ir by the atomic layer deposition method with the previously described process [11] using a Picosun SUNALE ${ }^{\mathrm{TM}} \mathrm{R} 150$ reactor. In order to verify the conformity of the deposited Ir layer we inspected local cross sections of the FZP, which was cut using a focused ion beam milling. As can be seen in Fig. 3, the Ir layer uniformly covers the sidewalls of a Si template.

Focusing experiments using the fabricated FZPs were performed in the X-ray scanning transmission microscope (STXM) at the PolLux beam line [12] of the Swiss Light Source (Villigen, Switzerland). The measurements were performed at $1 \mathrm{keV}$ x-ray energy. As a test object we used structures with periods down to $30 \mathrm{~nm}$ (i.e., $15 \mathrm{~nm}$ lines and spaces), prepared in a similar way as the zone plates. Figure 4(a) shows an STXM image of clearly resolved $15 \mathrm{~nm}$ line and space features. For comparison, a scanning electron microscope (SEM) image of the test structures is presented in Fig. 4(b). The measured DE of the FZP was $\sim 3 \%$, which is almost half of the theoretically expected DE. The remaining difference is mainly due to distortions in the sidewall shape of template lines and deviations of the linewidths in the template structure from the design (see Fig. 3).

In conclusion, we have developed a novel technique that frees us from the present limitations of electron-beam nanolithography and thereby improves the resolution of $\mathrm{x}$-ray microscopy by a factor of 2 . It enables the fabrication of high aspect ratio structures required for efficient ultrahigh-resolution x-ray optics. We are convinced that the results described do not represent the ultimate limitations of the method. It seems feasible to further increase the structure height, which would open up new possibilities for high resolution imaging in the multi-keV energy regime with elemental and chemical specificity. More importantly, it provides a clear route to push the resolution in soft x-ray imaging into the sub-10 nm regime. This would, in particular, give a possibility to resolve cytoskeletal elements in X-ray microscopy for the first time [13], representing an important step in cell biology research.

This work was partly funded by EU project "Integrating Activity on Synchrotron and Free Electron Laser Science Contract" under the Contract No. RII3-CT-2004-506008 part JRA3 and by Departament d'Educació i Universitats de la Generalitat de Catalunya i del Fons Social Europeu. We would like to thank Anja Weber (PSI) for assistance during substrate preparation, George Tzvetkov (PSI) for assistance during the beam time at the SLS, and Victor Callegari (EMPA) for help with the FIB cross section.

[1] G. C. Yin et al., Appl. Phys. Lett. 89, 221122 (2006).

[2] H. C. Kang et al., Phys. Rev. Lett. 96, 127401 (2006).

[3] W. L. Chao et al., Nature (London) 435, 1210 (2005).

[4] D. C. Flanders and N. N. Efremow, J. Vac. Sci. Technol. B 1, 1105 (1983).

[5] C. David et al., Microelectron. Eng. 84, 1172 (2007).

[6] M. Leskelä and M. Ritala, Angew. Chem., Int. Ed. 42, 5548 (2003).

[7] M. Young, J. Opt. Soc. Am. 62, 972 (1972).

[8] This kinematic approximation gives good results in the limit of large zone widths and short wavelengths. For the structure dimensions demonstrated in this Letter, dynamical effects already begin to play a role, as described in G. Schneider, Appl. Phys. Lett. 71, 2242 ( 1997); J. Maser, and G. Schmahl, Opt. Commun. 89, 355 (1992). However, a full treatment of these effects is very complex and beyond the scope of this Letter.

[9] J. Kirz, J. Opt. Soc. Am. 64, 301 (1974).

[10] B. L. Henke, E. M. Gullikson, and J. C. Davis, At. Data Nucl. Data Tables 54, 181 (1993).

[11] T. Aaltonen et al., J. Electrochem. Soc. 151, G489 (2004).

[12] U. Flechsig, AIP Conf. Proc. 879, 505 (2007).

[13] D. Scherfeld, J. Struct. Biol. 123, 72 (1998). 